\title{
Experimental investigation of beam-target neutron emission at the ELISE neutral beam test facility
}

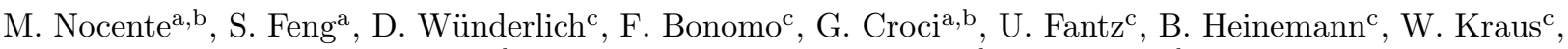 \\ I. Mario ${ }^{\mathrm{d}}$, R. Pasqualotto ${ }^{\mathrm{e}}$, M. Tardocchi ${ }^{\mathrm{b}}$, G. Gorini $^{\mathrm{a}, \mathrm{b}}$ \\ ${ }^{a}$ Dipartimento di Fisica "G. Occhialini", Università di Milano-Bicocca, Milano, Italy \\ ${ }^{b}$ Istituto di Fisica del Plasma, Consiglio Nazionale delle Ricerche, Milano, Italy \\ ${ }^{c}$ Max-Planck-Institut für Plasmaphysik, Garching, Germany \\ ${ }^{d}$ Università degli Studi di Padova, Padova, Italy \\ ${ }^{e}$ Consorzio RFX, Padova, Italy
}

\begin{abstract}
A dedicated experiment on beam-target neutron emission has been performed at the ELISE neutral beam test facility to investigate the accuracy of Local Mixing Model based calculations of neutron emission of relevance for the design of neutron diagnostics at the SPIDER neutral beam prototype. Compared to a previous experiment, we make use of fully developed infra-red and calorimetry diagnostics to include accurate data on beam profile and current in the calculations. Results show that variations of the neutron emission can be predicted with an accuracy better than 10\%, which compares to $30 \%$ in our previous investigation. At the same time, convincing evidence of diffusion effects beyond the Local Mixing Model is identified in the data. Implications of these findings for neutron emission at SPIDER are discussed.
\end{abstract}

Keywords: Beam-target neutron emission, Neutral beam injection, Neutron detectors PACS: [2010] 52.50.Gj, 52.70.Nc, 29.30.Hs

\section{Introduction}

In fusion plasmas, $2.5 \mathrm{MeV}$ neutron measurements are traditionally used to provide information of the fuel ion energy distribution in tokamaks 1, 2, 3. More recently, as the focus of fusion research progressively shifts from physics to technological applications, $2.5 \mathrm{MeV}$ neutron detectors using Gas Electron Multipliers (4) have been also proposed as diagnostics of the beam homogeneity for the SPIDER and MITICA Neutral Beam Injection Prototypes (NBI) in Padua. The detector exploits the beam-target neutron emission that occurs when the deuterium beam penetrates in the metallic dump of the NBI prototype.

In order to investigate the detailed physics behind this process and aid the detector design, in 2014 a parasitic experiment [5] was performed at the ELISE facility 6, 7, of the Max Planck Institut für Plasmaphysik in Garching. In this first study, a calibrated EJ301 liquid scintillator was used to measure neutron emission and benchmark calculations based on the Local Mixing Model (LMM) of deuterium implantation in the dump. The experimental results were generally found to be consistent 25 with calculations but, at a detailed level, an overestimation of up to $30 \%$ of the emission was often observed.

A dedicated experiment was performed at ELISE to clarify this discrepancy and is presented here. The new study makes use of fully developed infra-red and calorimetry diagnostics to provide more accurate input data for the calculations. Results are analysed as a test of the capability of the LMM to predict the magnitude of neutron emission in beam35 target reactions and to trace possible evidence of diffusion effects beyond the LMM in the data.

\section{Beam-target neutron emission experi- ment}

The neutron emission experiment we performed consists in a set of pulses at increasing value of the beam current (I) in the range $\mathrm{I}=5$ to $17 \mathrm{~A}$ and at a fixed total voltage $\mathrm{V}=30 \mathrm{kV}$. The beam optics 

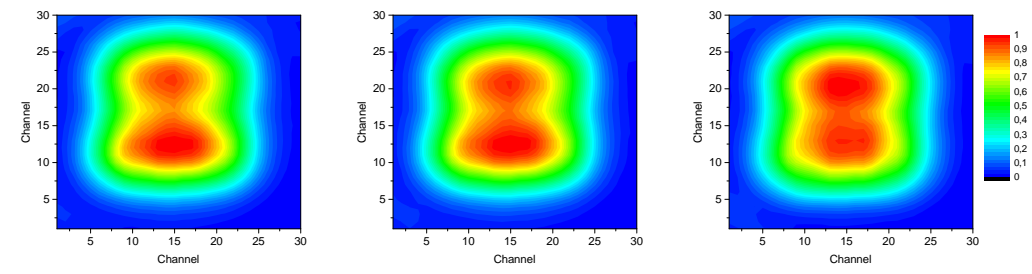

Figure 1: Beam profiles measured during the neutron emission experiment with the ELISE infra-red camera and normalised to unity. Measurements are shown at $\mathrm{t}=800,1200$ and $1600 \mathrm{~s}$ (from left to right). The times indicated here refer to those in figure 2

was carefully designed so to illuminate most of the dump surface and to achieve very similar beam pro-

45 files when the machine was operated at increasing values of the current (see figure 1). An infra-red (IR) camera was used to measure the beam profile in each of the discharges we performed. The profile was determined by an image of the infrared map 50 from the dump and which, after careful analysis, provides the power density distribution $\left(\mathrm{W} / \mathrm{m}^{2}\right)$ on the dump on a $30 \times 30$ grid.

Neutron emission from the dump was monitored by means of the same neutron detector used in our pre-

55 vious experiment [5], i.e. a calibrated EJ301 liquid scintillator produced by Scionix and coupled to a Hamamatsu H10580 photomultiplier tube. Signals from the liquid scintillator were recorded by a 14 bit, $400 \mathrm{MS} / \mathrm{s}$ digitiser based on the ATCA plat-

60 form [8, 9]. A standard neutron/gamma-ray separation algorithm was adopted to discriminate the 100 3 times more abundant neutrons from gamma-rays and with an estimated failure rate of $5.7 \%$, independently of the beam current.

In order to interpret data, for each of the pulse we performed, the expected time dependent neutron yield was determined based on the local mixing model (LMM) of deuterium deposition in the 105 dump made by $99 \%$ of copper. As in 5, we used the TRansport of Ion in Matter (TRIM) code 10 to evaluate the beam penetration in the dump. This was the input to calculate the local deuterium density $n_{D}(x, t)$, from which the neu- 110 tron yield $y(x, t)$ (neutrons $/ \mathrm{m}^{3} / \mathrm{s}$ ) is obtained as

$75 y(x, t)=n_{D}(x, t) \phi(x, t) \sigma$. Here, $x$ indicates an axis orthogonal to the dump surface. $\phi$ and $\sigma$ are the impinging deuterium flux and the (energy dependent) $2.5 \mathrm{MeV}$ neutron cross-section, respectively. As 115 more deuterium is implanted in the dump, the concentration $n_{D}(x, t)$ increases with time until a saturation level is reached ( $20 \%$ of total atoms based on our previous experiment). The total neutron emission $Y$, which is obtained by integrating $y(x, t)$ over the dump volume, depends on time, as it re5 flects the increasing deuterium concentration in the dump until it reaches the steady state condition. An essential parameter for the simulations is the impinging deuterium flux $\phi$, that is obtained from the current $I$, whose precise evaluation requires additional care. In our first experiment, we estimated the current reaching the dump by the total extracted current $I_{e x}$, i.e. the negative ion current that is extracted from the radio-frequency source. The accelerated current reaching the dump, $\mathrm{I}_{\mathrm{acc}}$, can be however different from $\mathrm{I}_{\text {ex }}$, up to about 20$30 \%$, as some ions are stopped by the grounded grid and the ground shield in their path from the radiofrequency source to the dump 11. An important improvement of our new experiment has been the capability to precisely evaluate the current reaching the dump, $\mathrm{I}_{\mathrm{acc}}$, thanks to water calorimetry measurements at ELISE 12 .

\section{Results}

Figure2 left shows the neutron counting rate during our experiment, which consisted of 170 pulses performed in 3 consecutive days after a few days of technical tests in the third deuterium campaign at ELISE. For each pulse we collected data for $10 \mathrm{~s}$ and evaluated the average counting rate per pulse shown in the figure. The $\mathrm{x}$ axis is the cumulated beam time, i.e. the effective time of beam operations, net of pauses. The current values that were actually delivered by the machine are shown in figure 2 right. We aimed in particular at performing measurements at $\mathrm{I}=7,12,15$ and $17 \mathrm{~A}$, where the first value corresponds to a region where $<10 \%$ discrepancies between calculations and data were found in our first experiment. In order to reach the highest currents we optimised Cesium conditioning 

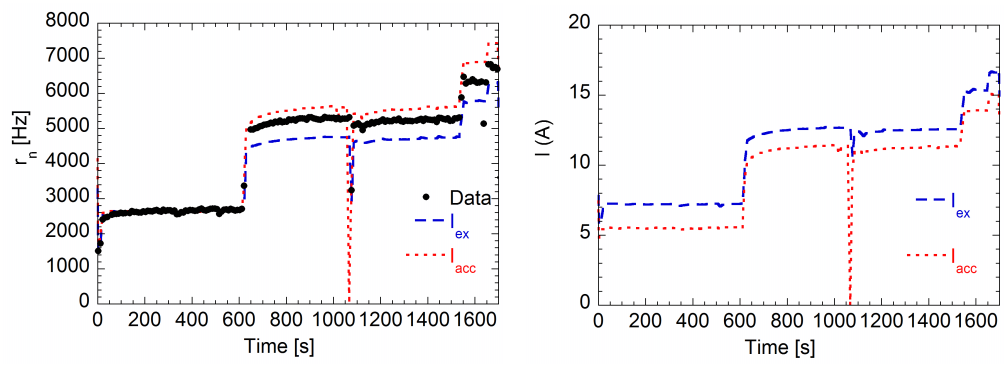

Figure 2: (left) Measured neutron rate in the experiment performed at ELISE. Data (dots) are compared to a calculation based on the LMM and using as input either the extracted ionic current ( $\mathrm{I}_{\mathrm{ex}}$, blue-dashed) or the accelerated current determined by calorimetry measurements $\left(\mathrm{I}_{\mathrm{acc}}\right.$, red-dotted). The error bars on the data are of the same size as the dots. (right) Values of $\mathrm{I}_{\mathrm{acc}}$ and $I_{e x}$ delivered by the machine during the experiment. The drop of $I_{\text {acc }}$ at $t \approx 1100$ s is due to a fault of the calorimeter diagnostic in one pulse.

of the radio-frequency source, the effect of which is seen, for example, in the moderately rising slope at $\mathrm{t}=600 \mathrm{~s}$ in figure 2 right. The acceleration voltage was always stable at $30 \mathrm{kV}$.

As neutron emission responds to changes of the curup of deuterium concentration in the dump, for each value of $\mathrm{I}_{\mathrm{ex}}$ we performed several pulses until a sufficiently stationary neutron rate was observed so to enable a clean comparison between data and simu- 16 lations. This is shown by the the dashed and dotted lines in figure 2 left, where calculation results based on $\mathrm{I}_{\mathrm{ex}}$ and $\mathrm{I}_{\mathrm{acc}}$ are displayed together. As the simulation provides the neutron emission $Y$ as a function of time, we need to evaluate the calibration coeffi- 170 cient $k$ that converts from $Y$ to the experimentally measured neutron rate $r_{n}$. Similarly to our previous experiment we have determined $k$ by the ratio between measurements and simulations using data points at $\mathrm{I}=7 \mathrm{~A}$, so that here the two simulation 175 is done, from the results of figure 2 we observe that, at $t>600 \mathrm{~s}$, calculations based on $\mathrm{I}_{\text {acc }}$ agree better with experimental results, as expected, showing that well calibrated neutron measurements are sen- 180 even to current variations smaller than $20 \%$, such as the difference between $\mathrm{I}_{\mathrm{ex}}$ and $\mathrm{I}_{\mathrm{acc}}$. As far as the beginning phase $(t<100 \mathrm{~s})$ at $\mathrm{I}=7 \mathrm{~A}$ is concerned, we note that our model is less accurate. This is due to some missing beam profile measure- ${ }_{185}$ ents during the technical discharges performed in the days preceding our experiment and is reflected in some uncertainties in our determination of the detailed build up of deuterium concentration before full saturation is reached (stationary neutron
A closer inspection to figure 2 however reveals that, albeit at the level of $<10 \%$, there still seems to be a systematic discrepancy between measurements and $\mathrm{I}_{\text {acc }}$ based simulations. This is better indicated by the simulation-to-experiment ratio in figure 3 , from which we note that $I_{a c c}$ based calculations tend to overestimate neutron emission. On the contrary, $\mathrm{I}_{\mathrm{ex}}$ based calculations always underestimate emission, but the agreement with measurements is improved as I gets larger. Compared to our former experiment, where we observed a disagreement at the level of $\approx 30 \%$ already at $\mathrm{I}=8$ to $10 \mathrm{~A}$, we can conclude that an accurate knowledge of the beam profile and current is essential for a reliable determination of the neutron emission and improves the agreement to a better than $10 \%$ level. The discrepancies observed earlier were most likely due to insufficient input diagnostic information. At the same time, however, we found no way to reconcile the remaining difference and we conclude that some additional deuterium diffusion caused by temperature effects away from the saturation state must be included in the model to completely account for the observations. This process, which is outside the LMM used here, does not play a very important role at the currents and voltages we have tested so far, as we were able to predict neutron emission with an accuracy better than $10 \%$. However, our data provide reasonable evidence that diffusion outside the LMM is at play in beam-target neutron emission experiments and becomes progressively more important as I is increased, as shown in figure 3 


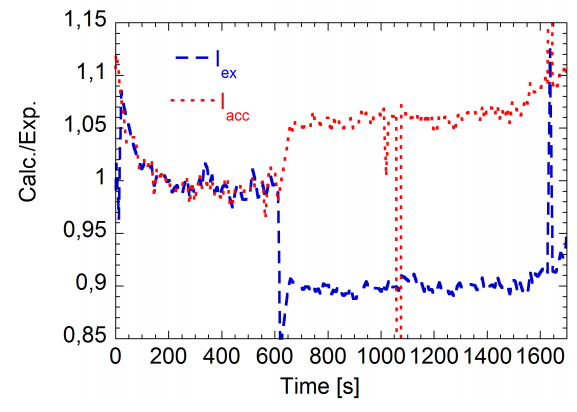

Figure 3: Ratio between neutron emission the calculations based on $\mathrm{I}_{\mathrm{acc}}$ and $\mathrm{I}_{\mathrm{ex}}$ and measured data of figure 2

\section{Discussion}

The experiment described in this paper was performed at beam current values $\mathrm{I}=7$ to $17 \mathrm{~A}$ and at a fixed acceleration voltage of $30 \mathrm{kV}$ in pulses lasting 10 seconds each. A maximum discrepancy of about $10 \%$ was observed between measurements and simulations once complete diagnostic information on the beam parameters were carefully ${ }^{245}$ included in the calculations under well controlled conditions. For comparison, SPIDER is meant to operate with $\mathrm{I}=40 \mathrm{~A}, \mathrm{~V}=100 \mathrm{kV}$, up to $1 \mathrm{~h}$ beam pulses and a twice larger beam dump. The power $I \cdot V$ delivered on the dump by the beam at SPIDER will therefore be about 8 times larger ${ }_{250}$ compared to our experiment. If we assume that deuterium diffusion outside the LMM scales as the beam power density, LMM based neutron emission calculations for SPIDER 13 may be overestimated by up to a factor 1.4. This suggests that a quantification of the diffusion coefficient that needs to be added to the neutron emission model is needed for reliable predictions at SPIDER. In this paper, we do not have data at $\mathrm{I}>17 \mathrm{~V}$ and $\mathrm{V}>30$ $\mathrm{kV}$ to aim at this quantification, but we limit ourselves to highlighting the parameters (beam profile, current on the dump) that are important ${ }^{260}$ for careful beam-target neutron calculations and to providing clear experimental evidence of the role of diffusion effects beyond the LMM in beam-target neutron emission. At the same time, we mention ${ }^{265}$ here that such quantification may be possible in future high power deuterium campaigns at ELISE if the machine is operated at parameters close to the maximum design performance, i.e. $\mathrm{I}=20 \mathrm{~A}$ and $\mathrm{V}=60 \mathrm{kV}$. This would correspond to a power on the dump of 1.2 MW, i.e. still a factor of 3 less than SPIDER, but also a factor 2 more than obtained at the largest currents of the present experiment, which is judged relevant to quantitatively study deuterium diffusion effects.

\section{Conclusions}

230

A dedicated experiment on beam-target neutron emission has been performed at the ELISE neutral beam test facility to investigate the accuracy of Local Mixing Model based calculations of neutron emission of relevance for the design of neutron diagnostics at SPIDER. Compared to a previous investigation, the experiment made use of fully developed beam current and profile diagnostics. We find that variations of the neutron emission can be described with an accuracy better than $10 \%$ at $\mathrm{V}=30 \mathrm{kV}$ and $\mathrm{I}=7$ to $17 \mathrm{~A}$, which compares to about $30 \%$ in the previous experiment where no accurate knowledge of the beam profile and current was available. The emission is mostly sensitive to variations of the current hitting the dump. At a more detailed level, the experimental results also reveal that diffusion effects beyond the Local Mixing Model are at play in beam-target reactions and may reduce neutron emission up to a factor 1.4 in full power operations at SPIDER compared to a previous evaluation.

\section{Acknowledgments}

The work leading to this publication was funded partially by Fusion for Energy under the Contract No. F4E-RFXPMS_A-WP-2016. The views and expression expressed herein do not necessarily reflect those of the Fusion for Energy and ITER organizations.

\section{References}

[1] M. Nocente, et al., Nucl. Fusion 54 (10) (2014) 104010.

[2] C. Cazzaniga, et al., Rev. Sci. Instrum. 85 (4) (2014) 043506.

[3] J. Eriksson, et al., Nucl. Fusion 55 (12) (2015) 123026.

[4] G. Croci, et al., JINST 7 (2012) C03010.

[5] X. Xufei, et al., Rev. Sci. Instrum. 85 (2014) $11 \mathrm{D} 864$.

[6] P. Franzen, et al., Nucl. Fusion 55 (2015) 053005.

[7] B. Heinemann, et al., Fusion Eng. Des. 84 (2009) 915.

[8] M. Nocente, et al., Rev. Sci. Instrum. 81 (2010) 10 D321.

[9] M. Nocente, et al., IEEE Trans. Nucl. Sci. 60 (2) (2013) 1408.

[10] J. Ziegler, SRIM/TRIM code, http://www.srim.org.

[11] P. Franzen, AIP Conf. Proc. 1655 (2015) 060001.

[12] R. Nocentini, et al., Fusion Eng. Des. 88 (6-8) (2013) 913

[13] M. Rebai, PhD

Thesis, 